\title{
EXCELLENT OUTCOME AFTER EARLY SURGERY FOR RUPTURED MIDDLE CEREBRAL ARTERY ANEURYSM COMPLICATED WITH INTRACEREBRAL AND INTRAVENTRICULAR HEMORRHAGE: A CASE REPORT
}

\author{
Venko Filipce $^{1}$, Aleksandar Caparoski ${ }^{1}$, Tomi Kamiloski ${ }^{1}$ and Dejan Daskalov ${ }^{2}$ \\ ${ }^{1}$ University Clinic for Neurosurgery, ${ }^{2}$ University Clinic for Radiology, University "Ss. Cyril and Methodius", \\ Medical Faculty, Skopje, Republic of Macedonia
}

\begin{abstract}
Intracerebral hematoma and intraventricular hemorrhage can significantly worsen the neurological condition of patients with subarachnoid hemorrhage due to ruptured intracranial aneurysm. They also significantly affect the outcome of this patiens. We present a case in which early surgery had a significant effect on the outcome of this patient. Early decompression of the brain and subsequent clipping of the aneurysm as presented in our case has lead to a successful outcome.
\end{abstract}

Key words: intracranial aneurysm, intracerebral hematoma, intraventricular hemorrhage

Correspondence to: Venko Filipce, University Clinic for Neurosurgery, "Vodnjanska" 17, 1000 Skopje, R. Macedonia; E-mail: venkofilipce@yahoo.com

\section{Introduction}

Intracerebral hematoma and intraventricular hemorrhage can significantly worsen the neurological condition as well as the outcome of patients with subarachnoid hemorrhage due to ruptured intracranial aneurysm [1-3].

\section{Case report}

We present a case of a 56-year-old female patient, who was admitted to our Clinic due to sudden transient loss of consciousness half an hour before the admission. During the neurological evaluation she was conscious, with stiff neck and nausea, no focal neurological deficit was present. According to Hunt and Hess scale patient was in grade was 2. Computed tomography (CT) scan
(Figure 1a) of the brain showed subarachnoid hemorrhage in the basal cisterns, dominantly in the right Sylvian fissure, compressive intracerebral hematoma in the temporal lobe region, midline shift of $4 \mathrm{~mm}$ and intraventricular hemorrhage. While still in the Emergency Room, neurological condition suddenly worsened and the patient fell in coma; pupils were normal and reactive to light and decerebration movements were bilaterally present. The patient stopped breathing. She was instantly intubated, and moved to Neurosurgical Intensive Care Unit for a respiratory support. Blood tests were done and the patient was prepared for surgical procedure. Half an hour after the worsening new CT revealed a larger intracerebral clot and a larger of blood in the ventricles. CT angiogram (Figure 1b) showed a large Middle Cerebral Artery (MCA) aneurysm with wide neck in which both branches were incorporated. The patient was successfully operated, hematoma was evacuated and the aneurysm was clipped. Postoperatively, (Figure 1c) she was sedated for a day after which sedation was ceased but due to agitation it was continued for four more days. The fifth day after discontinuation of the sedation the patient was awakened and extubated; she did not have neurological deficit.

\section{Discussion}

Aneurysmal intracerebral hematoma (ICH) is strongly associated with poorer functional outcome and seems to be predictable even before the bleeding event [1-3]. Aneurysms from distal anterior cerebral and middle cerebral artery are most likely to produce ICH and intraventricular hemorrhage (IVH) [4-6]. Intracerebral hematomas complicate $34 \%$ of aneurysm cases $[7,8]$. The sites of the hematoma depend on the direction of rupture of the aneurysm. Indication for angiography must be based on clinical suspicion. In our case we estimated that although the patient was in coma, additional imaging with CT angiogram had to be performed as soon as possible (in our center radiologist for angio- 
gram is on call after the working hours, i.e. not regularly present in the hospital). In a retrospective study [2] that included 132 patients with intracerebral hema- toma due to ruptured cerebral aneurysm, discriminant function analysis showed that, in order of importance,

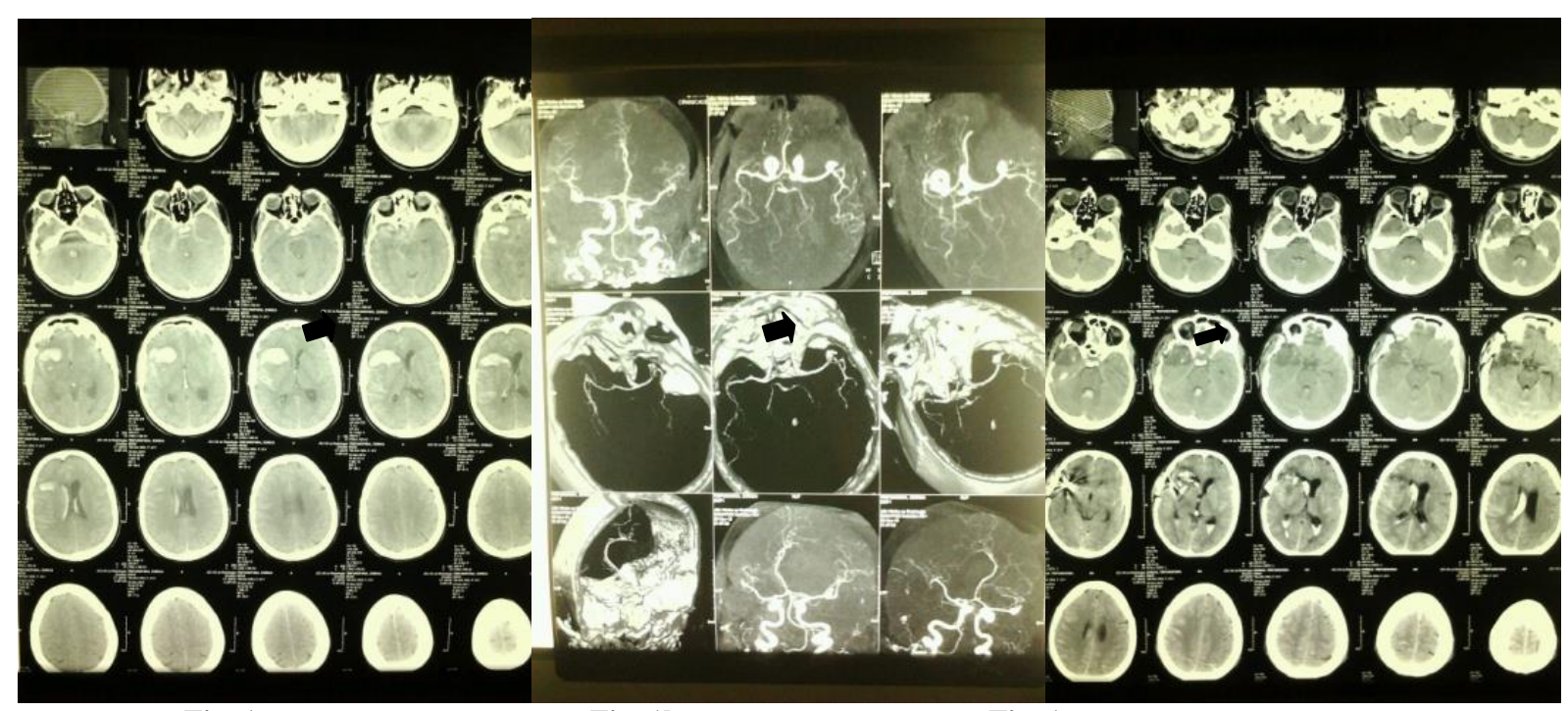

Fig. 1a.
Fig. 1c.

Legend: Figure 1a: initial CT scan presenting subarachnoid hemorrhage, intracerebral hematoma and intraventricular hemorrhage; Figure $1 \mathrm{~b}$ : CT angiography showing aneurysm on the right MCA at the level of the bifurcation. Figure 1c: postoperative CT scan with hipodensity at the site of evacuated intracerebral hematoma, intraventricular hemorrhage still present as expected. Arrows point out the intracerebral hematoma and aneurysm site.

size and location of the hematoma, aneurysm loocation and size of midline shift were factors contributing to prediction of survival. Hematoma size was also a strong predictor of clinical grade. About $40 \%$ of clots were frontal and $40 \%$ were temporal. Patients with temporal lobe clots have the greatest capacity of survival [9].

Our patient has shown an excellent outcome after surgery for ruptured MCA aneurysm with intracerebral hematoma and intraventricular hemorrhage. Although both factors significantly worsen the outcome, this case shows that if this complication of re-rupture appears while in hospital, fast/early surgery for clot evacuation and aneurismal clipping can result in good outcome and recovery after this type of devastating hemorrhage.

\section{Conclusion}

Intracerebral hematoma with intraventricular hemorrhage caused by ruptured intracranial aneurysm usually causes significant compression and destruction on the brain tissue. Midline shift caused by the compressive effect of the intracerebral hematoma is an indication for early surgery. Only early decompression of the brain and subsequent clipping of the aneurysm can decrease the mortality and morbidity from such devastating events to the brain.
In this case we are presenting our experience in which early surgery have significant effect on the good outcome in patients with compressive hematoma due to ruptured intracranial aneurysm.

Conflict of interest statement. None declared.

\section{References}

1. Kim HJ, Lee SW, Lee TH, Kim YS. Huge Intramural Hematoma in a Thrombosed Middle Cerebral Artery Aneurysm: A Case Report. J Cerebrovasc Endovasc Neurosurg 2015; 17(3): 234-238.

2. Nanda A, Ambekar S, Sharma M. Surgical management of middle cerebral artery aneurysms. J La State Med Soc 2014; 166(4): 160-167.

3. Zhao B, Cao Y, Tan X, et al. Complications and outcomes after early surgical treatment for poor-grade ruptured intracranialaneurysms: A multicenter retrospective cohort. Int J Surg 2015; 23(Pt A): 57-61.

4. Czorlich P, Ricklefs F, Reitz M, et al. Impact of intraventricular hemorrhage measured by Graeb and LeRoux score on case fatality risk and chronic hydrocephalus in aneurysmal subarachnoid hemorrhage. Acta Neurochir (Wien) 2015; 157(3): 409-415.

5. Fukuda H, Hayashi K, Moriya T, et al. Intrasylvian hematoma caused by ruptured middle cerebral artery aneurysms predicts recovery from poor-grade subarachnoid hemorrhage. J Neurosurg 2015; 123(3): 686-692. 
6. Jabbarli R, Reinhard M, Roelz R, et al. Intracerebral Hematoma Due to Aneurysm Rupture: Are There Risk Factors Beyond AneurysmLocation? Neurosurgery 2015 Nov 25. [Epub ahead of print]

7. Benoit RG, Cochrane DD, Durity F. Clinical-radiological correlates in intracerebral hematomas due to aneurismal rupture. Can J Neurol Sci 1982; 9(9):409-414.
8. Talamonti G, Nichelatti M, Al Mashni AA, D'Aliberti G. Life-threatening cerebral hematoma due to aneurysm rupture. World Neurosurg 2015 Sep 7. [Epub ahead of print]

9. Yokota H, Ida Y. CT angiography demonstration of ongoing intraventricular hemorrhage from actively bleeding posterior communicating artery aneurysm. Neurol India 2014; 62(4): 477-478. 